\title{
Images of Anomalous Origin of The Right Coronary Artery From the Pulmonary Artery
}

\author{
He Fan, Weng Jiakan, He Qicai, Qian Ximing, Chen Huaidong \\ Department of Cardiac Surgery, Sir Run Run Shaw Hospital, School of Medicine, Zhejiang University, Hangzhou, Zhejiang, \\ 310020, China
}

\section{ABSTRACT}

Anomalies of the coronary arteries -- especially their abnormal origin from the pulmonary artery (ARCAPA) trunk -- are among the least common. They're also the most dangerous of congenital heart defects with an incidence of $0.002 \%$ in the general population [Williams 2006]. The diagnosis exceedingly is difficult because anatomical abnormalities of the coronary arteries are subtle. We present a case of an anomalous origin of the right coronary artery.

\section{INTRODUCTION}

A 52-year-old male patient was referred to our hospital for gallbladder stones without symptoms associated with the cardiovascular system. Physical examination and X-ray revealed an enlargement of the cardiac boundary.

\section{RESULTS}

The patient's initial electrocardiography (ECG) demonstrated sinus tachycardia without acute changes. His troponin I index was normal. Computed tomography angiography (CTA) examination was then used for more detailed evaluation. As shown in Figure 1, the left and right coronary arteries and their branches markedly were tortuous and thickened. The right coronary artery had an anomalous origin from the pulmonary artery, and the diameters of the left and right coronary artery orifice considerably were enlarged. The right coronary artery markedly was tortuous with beaded changes in the crosssection view, and collateral circulation had formed between the right coronary and left anterior descending coronary artery.

Transthoracic echocardiogram indicated a normal function of the left and right ventricles, and the ejection fraction was estimated by the range of $64.5 \%$ to $70 \%$. There were no other

Received March 1, 2019; received in revised form fune 20, 2019 accepted fune 21, 2019.

Correspondence: Chen Huaidong, Department of Cardiac Surgery, Sir Run Run Shaw Hospital, School of Medicine, Zhejiang University, Hangzhou, Zhejiang, 310020, China; Tel: +8613817255875 (e-mail: $3317025 @ z j u . e d u . c n)$. structural cardiac abnormalities. The thickness of the left ventricular posterior wall and septal thickness was mildly increased. Multiple cardiac fistulas from the left and right coronary arteries ran into the right ventricular outflow tract, left atrium, left ventricle, and right atrium, respectively (Figure 2). The maximum flow rate of the right ventricular outflow tract was $2.7 \mathrm{~m} / \mathrm{s}$. The maximum pressure gradient (PGmax) was $29 \mathrm{mmHg}$, and the right ventricular systolic pressure was $35 \mathrm{mmHg}$.

\section{DISCUSSION}

When it comes to anomalous coronary artery disease, $22 \%$ of patients have other congenital heart anomalies, such as aortopulmonary window, tetralogy of Fallot, and ventricular septal defect (VSD) [Maluf 1997]. The diagnostic modalities for
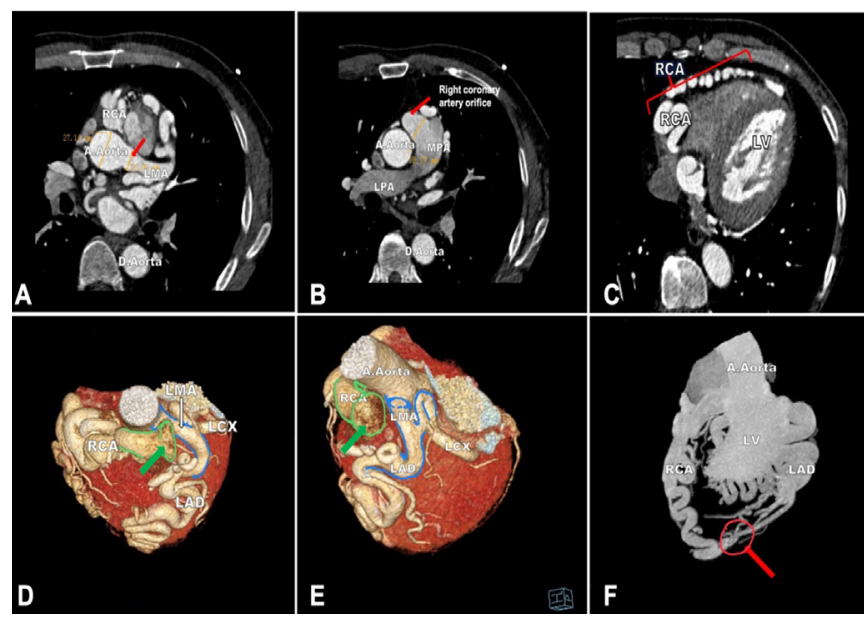

Figure 1. (A) CT coronary angiography of left main coronary artery (LMA) arises from the left aortic sinus and dilates; (B) Anomalous origin of the right coronary artery (RCA) from the pulmonary trunk (red arrow); (C) The right coronary arteries and their branches markedly were tortuous and thickened; (D) and (E) 3D computed tomographic reconstruction images showed the origin of left coronary artery and anomalous origin of right coronary artery (green arrow); (F) There is a collateral circulation between the terminal branches of the left and right coronary artery (red arrow). RCA, right coronary artery; LMA, left main coronary artery; A. Aorta, Ascending aorta; D. Aorta, Descending aorta; MPA, main pulmonary artery; LPA, left pulmonary artery; LV, Left ventricle; LAD, left anterior descending branch; LCX, left circumflex artery. (Shown by arrow) 


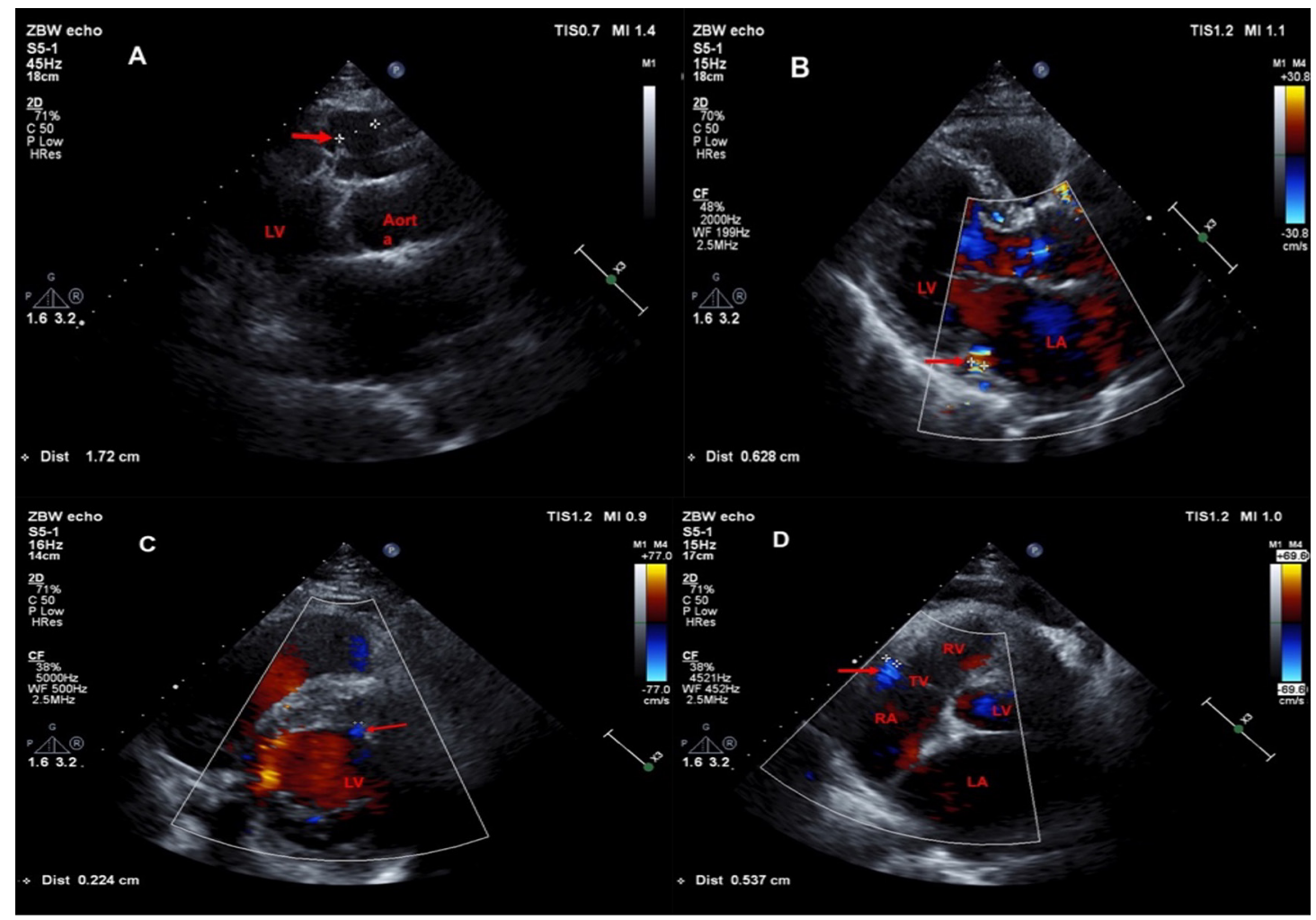

Figure 2. The views of transthoracic echocardiography. (A) A large fistula about $17.2 \mathrm{~mm}$ diameter was seen at the left anterior wall of the right ventricular outflow tract near the pulmonary valve ring; (B) The long axis view of aorta showed that a $6.3 \mathrm{~mm}$ diameter fistula was found in the left atrium near the mitral valve ring in the posterior wall of the left atrium; (C) Multiple abnormal blood flows were observed in the left ventricle, with a larger one about 2.2mm; (D) A 5.4mm wide fistula was observed on the right side wall of the right atrium near the tricuspid valve ring. $L A=$ left atrium; $L V=$ left ventricle; $R A=$ right atrium; $R V=$ right ventricle; TV, tricuspid valve. (Shown by arrow).

ARCAPA include cardiac computed tomography, cardiovascular magnetic resonance, and coronary angiogram, all of which comprise the Multi-Slice Computed Tomography (MSCT) coronary angiography, which is more reliable and plays an important role in accurate diagnosis. Patients with ARCAPA mostly are asymptomatic, but potentially life-threatening, surgical treatment of ARCAPA is highly recommended [DodgeKhatami 2002]. The aim of surgical corrections is to eliminate "coronary steal" and other coexisting cardiac malformations. Since the risk of sudden cardiac death decreases with age, symptomatic relief is the main goal for treatment [Kautzner 1996].

In our patient, there were no symptoms of severe "coronary steal" and no other heart anomalies. Therefore, the patient refused to undergo surgical treatment and decided to return for a timely outpatient follow-up.

\section{REFERENCES}

Dodge-Khatami A, Mavroudis C, Backer C L. 2002. Anomalous origin of the left coronary artery from the pulmonary artery: collective review of surgical therapy[J]. The Annals of Thoracic Surgery 74(3): 946-955.

Kautzner J, Veselka J, Rohac J. 1996. Anomalous origin of the right coronary artery from the pulmonary trunk: is surgical reimplantation into the aorta a method of choice. Clin Cardiol. 19(3): 257-9.

Maluf MA, Smith M, Abellan DM, Troster EJ, Takaoka F, Rati M, et al. 1997. Anomalous origin of right coronary artery from the pulmonary artery in association with a ventricular septal defect. Tex Heart Inst J. 24:226-9.

Williams I A, Gersony W M, Hellenbrand W E. 2006. Anomalous right coronary artery arising from the pulmonary artery: a report of 7 cases and a review of the literature[J]. American heart journal 152(5): 1004. e9-1004. e17. 